\title{
Slippery Surface with Petal-like Structure for Protecting Al Alloy: Anti-corrosion, Anti-fouling and Anti-icing
}

\author{
Junfei Huang ${ }^{1,3} \cdot$ Jiajie Kang ${ }^{1,4} \cdot$ Jiaxu Zhang ${ }^{1,3} \cdot$ Jinxia Huang ${ }^{3} \cdot$ Zhiguang Guo $^{2,3}$
}

Received: 21 May 2021 / Revised: 29 October 2021 / Accepted: 14 November 2021 / Published online: 20 December 2021

(c) The Author(s) 2021

\begin{abstract}
The harsh working environment affects the performance and usage life of $\mathrm{Al}$ and its alloys, thus limiting their application. In recent years, Slippery Liquid-infused Porous Surface (SLIPS) has attracted much attention due to excellent anti-corrosion, anti-fouling and anti-icing properties. This may be an effective way to improve the properties of $\mathrm{Al}$ and its alloys. Here, the SLIPS with petal-like structure was constructed on the Al alloy via simple hydrothermal reaction, Stearic Acid (STA) modification and lubricant injection. A variety of droplets (including oil-in-water emulsions) can slide on the SLIPS at a low angle, even the Sliding Angle (SA) of the water droplet is only $3^{\circ}$. Furthermore, the SLIPS exhibits outstanding mechanical and chemical properties. It can maintain fine oil-locking ability under high shearing force and keep slippery stability after immersion in acid/alkaline solutions. In addition, the SLIPS possesses excellent anti-corrosion, anti-fouling and anti-icing properties, which provides a new way to promote the application of $\mathrm{Al}$ and its alloys. Therefore, the SLIPS is expected to be an effective way to improve the properties of $\mathrm{Al}$ and its alloys, as well as play a role in anti-fouling and self-cleaning in construction, shipbuilding and automotive manufacturing industries, thereby expanding the practical application of $\mathrm{Al}$ and its alloys.
\end{abstract}

Keywords Slippery surface $\cdot \mathrm{Al}$ alloy $\cdot$ Anti-corrosion $\cdot$ Anti-fouling $\cdot$ Anti-icing

\section{Introduction}

As one of the commonly used engineering materials, $\mathrm{Al}$ and its alloys have excellent physical and mechanical properties, making $\mathrm{Al}$ and its alloys widely used, especially in

Jiajie Kang

kangjiajie@cugb.edu.cn

$\triangle$ Jinxia Huang huangjx@licp.cas.cn

$\triangle$ Zhiguang Guo zguo@licp.cas.cn

1 School of Engineering and Technology, China University of Geosciences, Beijing 100083, China

2 Ministry of Education Key Laboratory for the Green Preparation and Application of Functional Materials, Hubei University, Wuhan 430062, China

3 State Key Laboratory of Solid Lubrication, Lanzhou Institute of Chemical Physics, Chinese Academy of Sciences, Lanzhou 730000, China

4 Zhengzhou Institute, China University of Geosciences (Beijing), Zhengzhou 451283, China aerospace, automotive, machinery manufacturing, shipbuilding and chemical industry. Therefore, it is inevitable for them to work in harsh environment, which will cause great damage to them and affect their usage life. To improve the usage life of $\mathrm{Al}$ and its alloys and expand its application range, it should be put on agenda that improving the properties of $\mathrm{Al}$ and its alloys under extreme environments [1-5]. In recent years, it has been considered as a new and effective way to construct Superhydrophobic Surfaces (SHSs) on $\mathrm{Al}$ and its alloys for prevent corrosion [6,7]. In addition, SHSs has attracted much attention in the fields of oil-water separation [8,9], anti-icing [10-12], self-cleaning [13, 14] and drag reduction [15] due to its excellent hydrophobic performance (contact angle greater than $150^{\circ}$ ). However, reports have shown that the instability of the hydrophobic layer and the finiteness of the micro-nano structure led to the insufficient robustness of SHSs, which makes them easy to be destroyed under dynamic action and mechanical damage $[16,17]$. Hence, the effect of building superhydrophobic surface on $\mathrm{Al}$ and its alloys to improve their performance is very limited. 
It is well known that Nepenthes pitcher plants can capture insects through the slippery surface. The reason for the formation of slippery surface is that the surface has a hydrophilic component and the rough structure, so the surface can store water and form a lubricating water layer [18]. Inspired by this, Aizenberg et al. proposed the concept of Slippery Liquid-infused Porous Surface (SLIPS). They injected lubricating oil into the rough porous surface to form SLIPS, which showed excellent characteristics including but not limited to low sliding angle, extreme pressure stability and optical transparency [19]. Surprisingly, there are some references pointing out that SLIPS has excellent repeatable self-healing ability [19-21]. Hence, SLIPS shows better mechanical durability and robustness than SHS, and SLIPS is expected to be an effective way to solve SHS failure. Up to now, SLIPS has become a hot research field, which has attracted the attention of many scientists. Based on the three principles followed in constructing SLIPS: (1) the lubricating liquid and the repellent liquid are not mutually soluble, (2) compared with the repellent fluid, the lubricating fluid must have higher chemical affinity with the substrate, and (3) the solid surface must be a nano-composite rough structure to provide large enough surface to absorb and store lubricating oil [19], many SLIPSs have been made by researchers and used in various fields. Sun et al. fabricated a stable superoleophobic-superhydrophilic surface by spraying, which can separate organic liquid mixtures [22]. Feng et al. developed a SLIPS with hierarchical Micro-Nanostructures (MNS-SLIPS) on the basis of regular microporous structures for long-term efficient water harvesting [23]. Li et al. fabricated a slippery copper oxide surface by a simple liquid-solid reaction, STA modification and oilinfused, the surface inhibits corrosion efficiency reached up to $92.68 \%$ [24]. Therefore, building SLIPS on Al and its alloys may be a promising potential method to improve its performance and expand its application.

In this work, we have prepared the SLIPS with petallike structure on $\mathrm{Al}$ alloy substrate by simple hydrothermal method, with low surface energy modification and lubricant injection. The lubricant was locked in the rough structure of the SHS to form the SLIPS, so the water droplet can easily slide on the SLIPS with a small Sliding Angle (CA) about $3^{\circ}$. And the SLIPSs showed excellent mechanical and chemical stability, whether under high shear condition or in acidic/alkaline solutions. Furthermore, we conducted a series of tests on the samples, and the results showed that compared with the original surface and SHS, SLIPS had better anti-corrosion, anti-fouling and anti-icing properties. Therefore, building SLIPS on the Al alloy substrate can improve its performance, which is of great help to expand its practical application and extend its life.

\section{Experiments}

\subsection{Materials}

The Al substrates (AA6061 alloy) were purchased from Shanghai Haocheng Metal Co. Ltd. China, and they were cut into $2 \mathrm{~cm} \times 2 \mathrm{~cm}$ pieces. Stearic Acid $\left(\mathrm{STA}, \mathrm{CH}_{3}\left(\mathrm{CH}_{2}\right)_{16} \mathrm{COOH}\right)$ purchased from Guangdong Province Fine Chemical Engineering Technology Research and Development Center. Zinc nitrate hexahydrate $\left(\mathrm{Zn}\left(\mathrm{NO}_{3}\right)_{2} \cdot 6 \mathrm{H}_{2} \mathrm{O}, 99.0 \%\right)$ was obtained from Tianjin Fangda Chemicals Co. Ltd. Ammonium nitrate $\left(\mathrm{NH}_{4} \mathrm{NO}_{3}\right)$ was supplied from BEIJINGSHIHONGXINGHUAGONGCHANG, China. Ammonia $\left(\mathrm{NH}_{3} \cdot \mathrm{H}_{2} \mathrm{O}\right)$, Dimethyl silicone oil (H201-100), and $\mathrm{NaOH}$ were all of analytical grade, and were supplied by Sinopharm Chemical Reagent Co. Ltd. Ethanol was purchased from Lianlong Bohua (Tianjin) Medical Chemicals Co., Ltd. All the reagents were of analytical grade and used as received without further purification. Deionized Water (DI) was used throughout the experiment.

\subsection{Preparation of Slippery Surface}

The samples were prepared based on the previous method [25]. Firstly, the $\mathrm{Al}$ alloy plates were sequentially abraded with emery sand paper of grades 800,1200 , and 1500 , cleaned ultrasonically in ethanol and DI water successively. The pretreated sample was named as Bare. To remove the oxide film on the Bare surface, the abraded $\mathrm{Al}$ alloy plates were etched in $0.1 \mathrm{M} \mathrm{NaOH}$ for $120 \mathrm{~s}$ at room temperature. And then the samples were ultrasonically cleaned with DI water and dried in air.

Secondly, the Bare was vertically immersed in a mixed solution of $0.05 \mathrm{M} \mathrm{Zn}\left(\mathrm{NO}_{3}\right)_{2}$ and $0.3 \mathrm{M} \mathrm{NH}_{4} \mathrm{NO}_{3}$. The $\mathrm{pH}$ was controlled to 6.5 by $1.0 \mathrm{wt} \% \mathrm{NH}_{3} \cdot \mathrm{H}_{2} \mathrm{O}$. The hydrothermal reaction was performed under hydrothermal treatment $\left(85^{\circ} \mathrm{C}\right.$, $12 \mathrm{~h})$. After that, the samples were washed by ethanol and dried in air naturally. The sample obtained by hydrothermal process is named as Layered Double Hydroxide (LDH).

Thirdly, the prepared samples were immersed in $0.05 \mathrm{M}$ STA/ethanol at $60^{\circ} \mathrm{C}$ for $6 \mathrm{~h}$ and then dried in air at room temperature. The obtained samples were denoted as SHS.

Finally, about $50 \mu \mathrm{L}$ dimethyl silicone oil was used to lubricate the as-prepared SHS. On the spin coater, spun coating at $500 \mathrm{rpm}$ for $30 \mathrm{~s}$ to spread the lubricant evenly on the SHS, and then threw out the excess lubricant at $1000 \mathrm{rpm}$ for $30 \mathrm{~s}$. And the final product was labelled as SLIPS.

\subsection{Mechanical and Chemical Stability Tests}

The mechanical and chemical stability test of the samples was tested into three parts. (i) In the lubricant retention capacity test, we compared the oil-locking ability of the LDH and SLIPS, samples (LDH/oil, SLIPS) were rotated 
at different spin rates ranging from 1000 to $5000 \mathrm{rpm}$ for $10 \mathrm{~s}$ to detect the mass variations. (ii) The CAs and SAs of droplets with different $\mathrm{pH}$ (pH from 1 to 14) on the sample surfaces were measured. (iii) The CAs and SAs of the samples were measured after immersion in $0.1 \mathrm{M} \mathrm{HCl}$ and $\mathrm{NaOH}$ solution for 10 min to explore the chemical stability of the samples.

\subsection{Electrochemical Corrosion Test}

The corrosion resistance of the samples was determined by three-electrode electrochemical workstation with 3.5 vt $\% \mathrm{NaCl}$ aqueous solution as electrolyte at room temperature. The platinum plate was used as the counter electrode, CHI150 Saturated Calomel Electrode (SCE) as the reference electrode, and the working electrode was the prepared sample. The polarization curves were obtained at the scanning rate of $5 \mathrm{mV} \cdot \mathrm{s}^{-1}$ from -0.3 to $3 \mathrm{~V}$ versus the open circuit potential. The parameters of corrosion potentials (Ecorr) and corrosion current density (Icorr) were obtained by Tafel extrapolation method. Each sample was measured at least three times.

\subsection{Anti-fouling and Anti-icing Experiments}

In the anti-fouling experiment, we selected several common drinks (including milk, tea, coffee and cola) to test the antifouling ability of the samples. Test at room temperature with the liquid evenly stirred.

During the anti-icing experiment, about $15 \mathrm{~mL}$ of water was put into the culture dish, the treated side of different samples (Bare, SHS and SLIPS) contacted with the water surface, and then the culture dish was placed in the refrigerator at $-10{ }^{\circ} \mathrm{C}$ to observe the changes of the sample surfaces.

\subsection{Characterization}

All sample morphologies were characterized by fieldemission Scanning Electron Microscope (SEM, QUANTA
FEG 650). The apparent Contact Angles (CAs) and Sliding Angles (SAs) were measured at room temperature by a JC2000D goniometer with a $10 \mu \mathrm{L}$ droplet. The chemical composition was analyzed via Fourier Transform Infrared spectroscopy (FTIR, Thermo Scientific Nicolet iS10). X-ray Photoelectron Spectroscopy (XPS, Thermo Scientific ESCALAB 250Xi) was used to characterize the quantitative elemental composition. The contents of the chemical elements were characterized via Energy Dispersive Spectroscopy (EDS, JSM-5600LV). The surface roughness of the samples was measured on a 3D non-contact surface profiler (KLA-Tencor MicroXAM-800).

\section{Results and Discussion}

\subsection{Surface Characterization}

In this work, we constructed SLIPS with petal-like structure on $\mathrm{Al}$ alloy substrate by a simple hydrothermal method. The manufacturing process was shown in Fig. 1. In brief, the pretreated $\mathrm{Al}$ alloy was immersed in the mixed solution of $0.05 \mathrm{M} \mathrm{Zn}\left(\mathrm{NO}_{3}\right)_{2}$ and $0.3 \mathrm{M} \mathrm{NH}_{4} \mathrm{NO}_{3}$, then the $\mathrm{pH}$ of the solution was adjusted to 6.5 , the reaction was carried out in an autoclave at $85^{\circ} \mathrm{C}$ for $12 \mathrm{~h}$. At this time, the surface of $\mathrm{Al}$ alloy was covered with $\mathrm{LDH}$ film. And then the sample was modified with ethanol/STA solution at $60{ }^{\circ} \mathrm{C}$ for $6 \mathrm{~h}$ to obtain SHS. Finally, dimethyl silicone oil was injected into the SHS to obtain SLIPS.

As we all know, the construction of superhydrophobic surfaces requires a certain surface roughness and low surface energy. In this work, the LDH film was constructed on aluminum alloy by hydrothermal reaction. After modification with stearic acid, carboxyl groups and metal ions form complexes on the surface [26], which further increased the roughness of the surface and reduced the surface energy to obtain the superhydrophobic surface, as shown in Fig. S1. Besides, we characterized the 3D surface profiles of the samples and measured their roughness. The surface roughness of
Fig. 1 Schematic illustration of the formation of the SLIPS

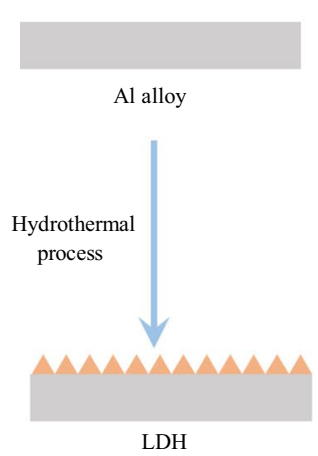

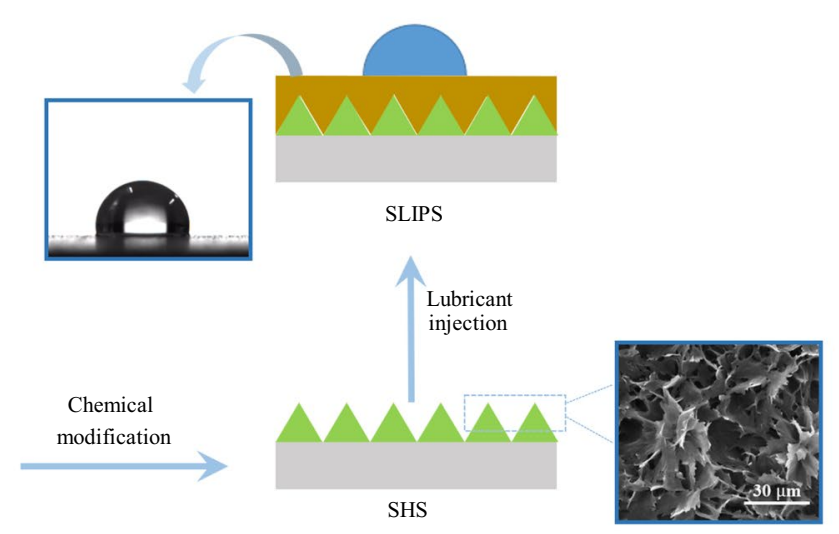


Bare, $\mathrm{LDH}$ and SHS are $0.018 \mu \mathrm{m}, 0.175 \mu \mathrm{m}$ and $1.63 \mu \mathrm{m}$, respectively (Fig. S2). The results show that STA modification has great influence on the surface morphology, increases the surface roughness of the sample and provide enough space for oil injection.

The Fig. 2a-d shown the SEM and water contact angle images of the samples under different processes. Figure 2a is the surface of the $\mathrm{Al}$ alloy after pretreatment, we can observe that there are scratches left by sandpaper rubbing on it. And the surface of bare $\mathrm{Al}$ alloy is hydrophilic with a water contact angle of $58^{\circ}$. After the hydrothermal reaction, the $\mathrm{Al}$ alloy surface is covered with LDH film. The water contact angle increases with the increase of surface roughness, but it still shows hydrophilicity. The water contact angle of the LDH film is $83^{\circ}$ (Fig. 2b). Figure $2 \mathrm{c}$ and d show the LDH modified by ethanol/STA solution at different magnification. We can clearly see the petal-like structures on the $\mathrm{Al}$ alloy surface in Fig. 2d. As shown in Fig. 2c, after modified with ethanol/STA solution, the water contact angle of the sample is about $155^{\circ}$, showing excellent superhydrophobicity. Since the surface tension of silicone oil is lower than that of water, silicone oil is easier to wet on superhydrophobic surfaces. When silicone oil was injected into the SHS, an oil film can be formed on the surface, and the petal-like structure of the SHS can lock the lubricating oil to reduce its loss, thus ensuring the stability of the SLIPS.

The water contact angle of SLIPS is $100^{\circ}$ and the water sliding angle is $3^{\circ}$ (Fig. 2e and f). The dynamic behavior was taken every $15 \mathrm{~s}$. In addition, to explore the smooth characteristics of the SLIPS, we also measured the CAs and SAs of different liquids including oil-in-water on the SLIPS (Fig. S3). It turns out that liquid not limited to water (N-hexanein-water, Oleic acid, Glycol, Ethanol and Diesel-in-water) can slide at a low SA on SLIPS. The above results are mainly attributed to the continuous lubricant layer of SLIPS, indicating that SLIPS has excellent smoothness.
The FTIR spectrum of samples are shown in Fig. 3a, due to the stretching and bending vibrations of hydroxyl groups, the broad peaks at 3402 and $1639.4 \mathrm{~cm}^{-1}$ appear on the spectrum [27]. The peaks at 1332.7 and $821.45 \mathrm{~cm}^{-1}$ are from $\mathrm{NO}_{3}{ }^{-}$, indicating that the LDH film is successfully formed on the $\mathrm{Al}$ alloy substrate after hydrothermal reaction. At 2916 and $2846.8 \mathrm{~cm}^{-1}$, SHS has the stretching vibration bands of $\mathrm{C}-\mathrm{H}$ [28]. And the peaks that occurred at 1537.2 and $1396 \mathrm{~cm}^{-1}$ are due to the asymmetric and symmetric vibrations of $\mathrm{COO}$ - groups [29]. According to the analysis of the FTIR, it can be concluded that modification of LDH film by STA has achieved nice results. As illustrated in Fig. 3b and c, the chemical compositions were also analyzed by the XPS spectrum to verify the element types of on the SHS. As shown in the Fig. 3b, the Al peak is not displayed, probably because $\mathrm{Al}$ is covered by other elements or the $\mathrm{Al}$ peak intensity is too weak. Figure $3 \mathrm{c}$ is the $\mathrm{C} 1 \mathrm{~s}$ spectra. The fitted peaks at $284.7,285.1$, and $288.7 \mathrm{eV}$ belonged to $-\mathrm{CH}_{2},-\mathrm{CH}_{3}$ and $\mathrm{O}=\mathrm{C}-\mathrm{O}$ groups of the STA $[25,30]$. These results further demonstrate that the hydrophobic groups of STA are grafted into LHD films to form SHS. Furthermore, the EDS results indicated that SHS has high content of C element (Fig. S4). All the above results indicate that SHS is obtained after STA modification, which lays the foundation for the fabrication of SLIPS.

\subsection{Chemical and Mechanical Properties}

The chemical and mechanical properties are important criteria to evaluate the quality of materials. Which has a significant impact on whether the material can be can be mass-produced and applied [31]. In practical applications, metal materials are inevitably used in harsh environments, so stable chemical and mechanical properties are extremely important for Al-based SLIPS. Therefore, we explored the effects of high shear force on the oil storage capacity of
Fig. 2 SEM images of a Bare, $\mathbf{b}$ LDH, c SHS, d high magnification SHS, e the CA of SLIPS and $\mathbf{f}$ dynamic behavior of a water droplet on the SLIPS

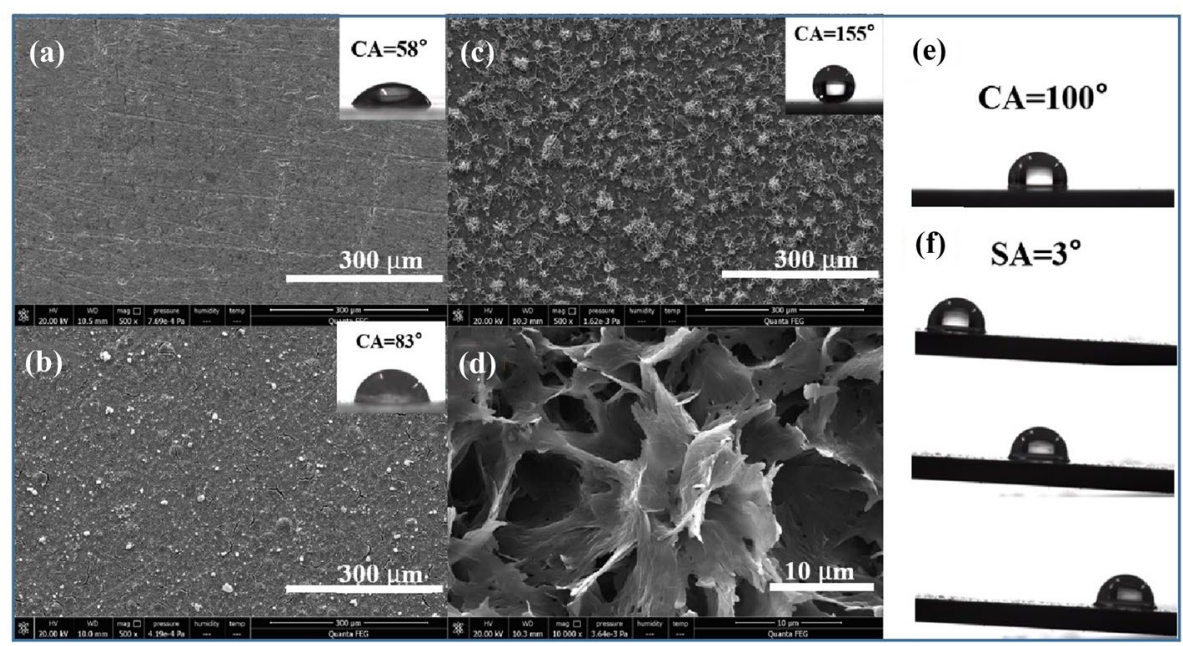


(a)

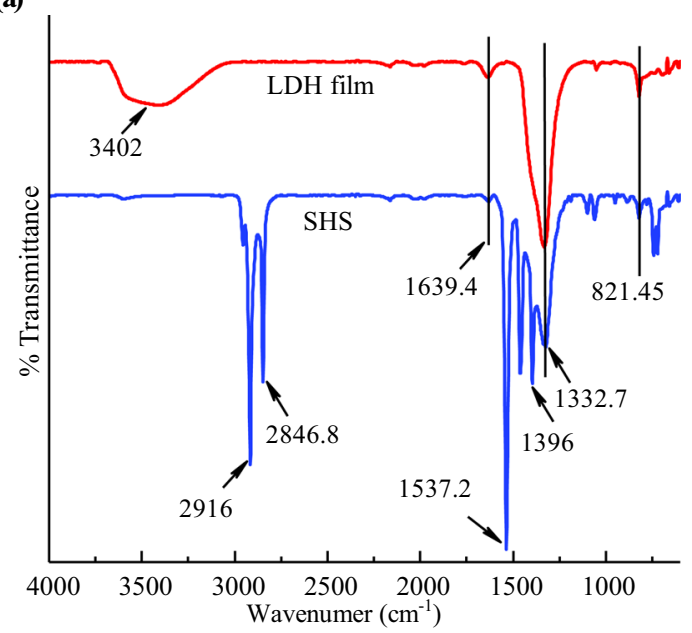

(b)

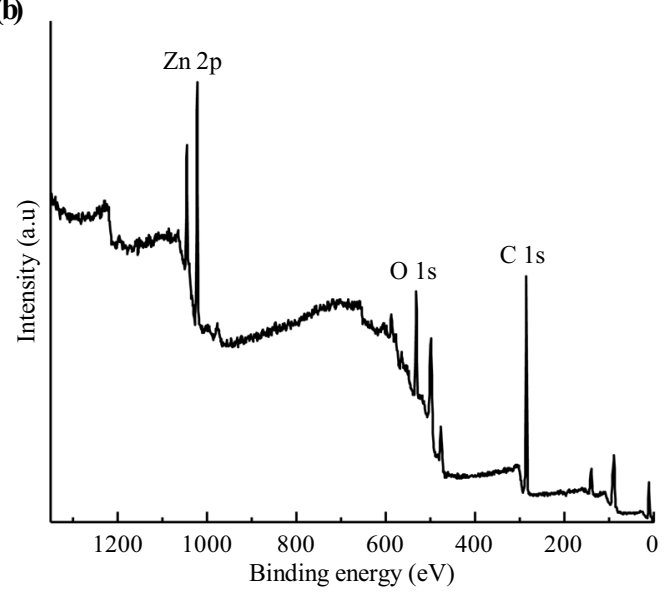

(c)

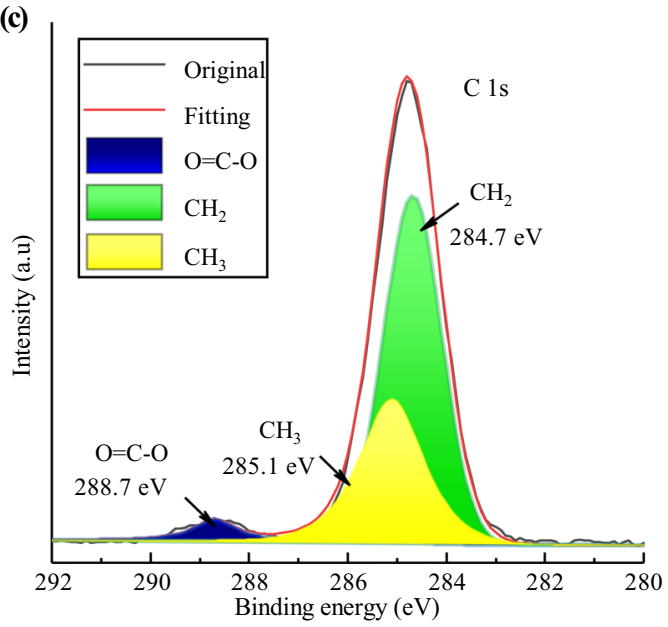

Fig. 3 a FTIR spectra of the LDH film and SHS, b XPS and c C $1 \mathrm{~s}$ spectra of SHS

samples and different $\mathrm{pH}$ solutions, acid and alkaline solutions on SLIPS.

For the exploration of the mechanical properties of the SLIPS, we mainly focus on oil retention capacity. To apply
Table 1 The initial condition of the samples

\begin{tabular}{lll}
\hline Samples & Initial weight $(\mathrm{g})$ & $\begin{array}{l}\text { Silicone } \\
\text { oil-infused } \\
(\mathrm{g})\end{array}$ \\
\hline LDH & 1.0133 & 1.0241 \\
SLIPS & 1.0176 & 1.0534 \\
\hline
\end{tabular}

a shearing stress on the samples, LDH/oil and SLIPS were placed on a spin-coater and rotated at different spin rates ranging from 1000 to $5000 \mathrm{rpm}$ for $10 \mathrm{~s}$ to detect the variations in weights. Then we test the oil-locking ability of the samples by the amount of residual silicone oil on the surfaces $(\Delta m=$ quality after centrifugal - Initial weight). The initial condition of the samples was shown in Table 1 and the test results were shown in Fig. 4a. According to the Table 1, after the completion of silicone oil injection, the average weight of SLPS increased by about $0.0358 \mathrm{~g}$ and LDH gained about $0.0108 \mathrm{~g}$. We preliminarily believe that the oil locking ability of slips is stronger than that of LDH. And then we measured the quality of the samples at different spin rate, it is clear that the amount of oil retained on the SLIPS is much higher than that of the LDH at different speeds. Finally, after $5000 \mathrm{rpm}$, LDH only retained about $18.44 \%$ of the oil while the SLIPS kept about $43.06 \%$. The results indicated that SLIPS showed preferable lubricant retention ability relative to $\mathrm{LDH}$, which can be interpreted from the point of surface morphology and structure, as mentioned above.

As shown in Fig. 4b, the contact angle of droplets with different $\mathrm{pH}$ value on the SLIPS changes little. This result shows that the oil film on the slippery surface has nice resistance to $\mathrm{H}^{+}$and $\mathrm{OH}^{-}$. This is mainly due to the stable chemical properties of silicone oil. With the increase of the concentration of $\mathrm{H}^{+}$and $\mathrm{OH}^{-}$, the sliding angle of water droplets on the SLIPS also increased slightly, but the sliding angle was still less than $10^{\circ}$. In general, the oil film on the slippery surface can prevent ions in the solution from destroying the substrate surface, so contact angle and sliding angle of the SLIPS do not change much under different $\mathrm{pH}$ values [32]. To further verify the chemical stability of the SLIPS, we immersed the SLIPS and SHS in $0.1 \mathrm{M} \mathrm{HCl}$ and $\mathrm{NaOH}$ solutions respectively for different times to observe the changes. In acidic and alkaline environments, the change results of CA and SA between the SLIPS and SHS were shown in Fig. 4c, $d$ and Fig. S5a. With the increase of immersion time, the CA of SHS decreased gradually, whether in acid solution or alkaline solution. Moreover, the CA of SHS decreased more rapidly in acidic environment, the reason may be that the surface of SHS is composed of layered double hydroxides, and LDH is easier to combine with hydrogen ion than hydroxyl ion. Fig. S5b shows the picture of the SHS after 
(a)

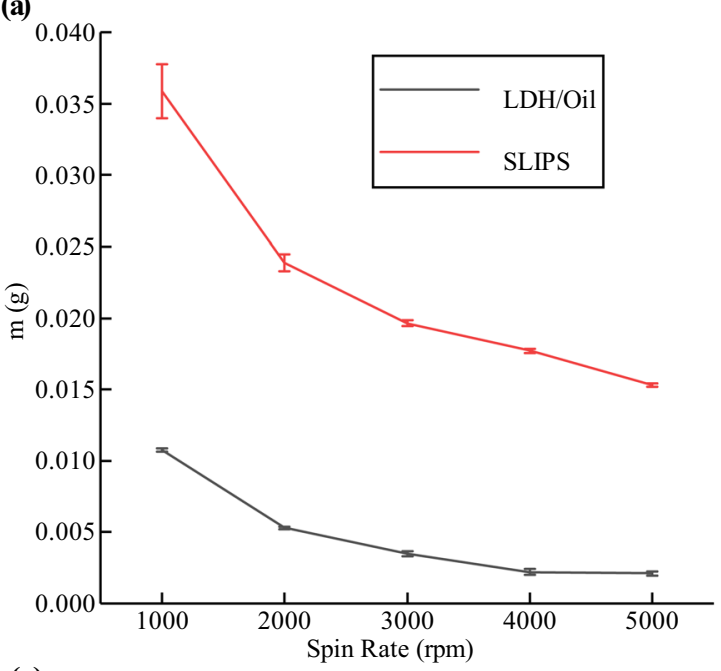

(c)

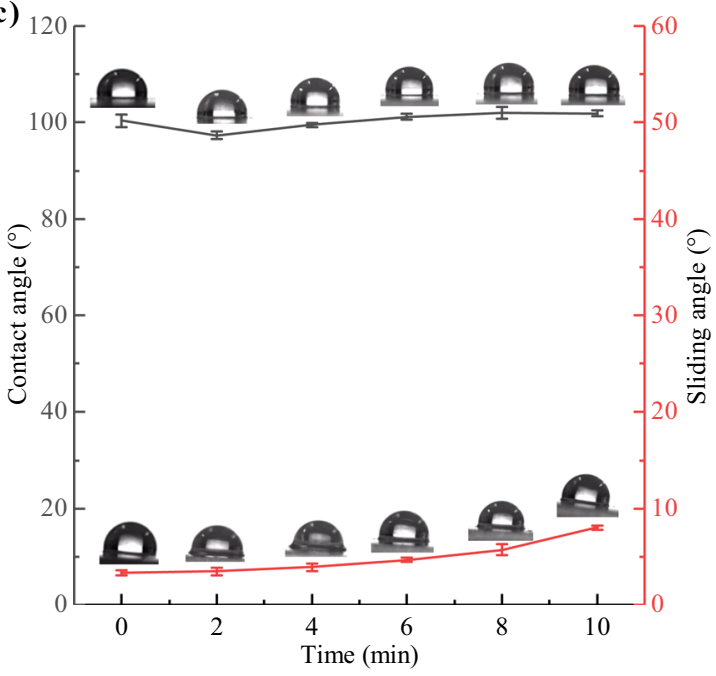

(b)

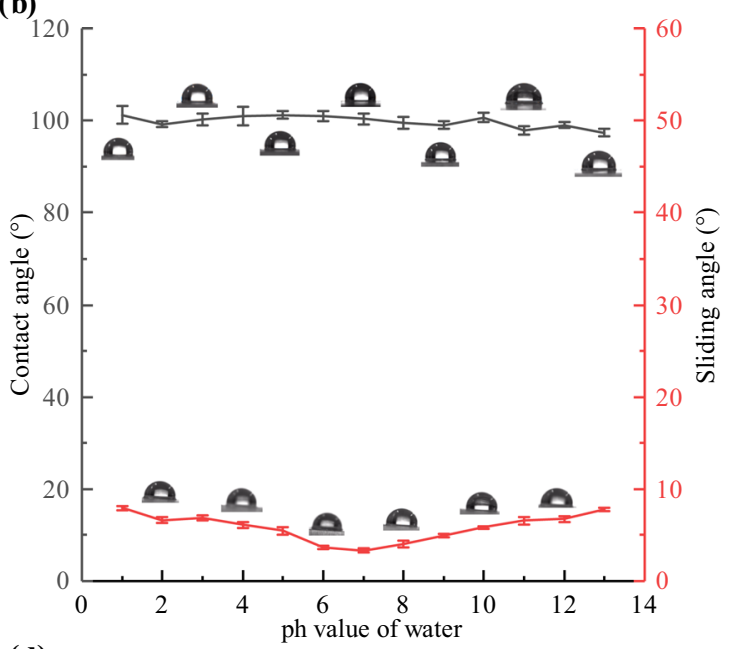

(d)

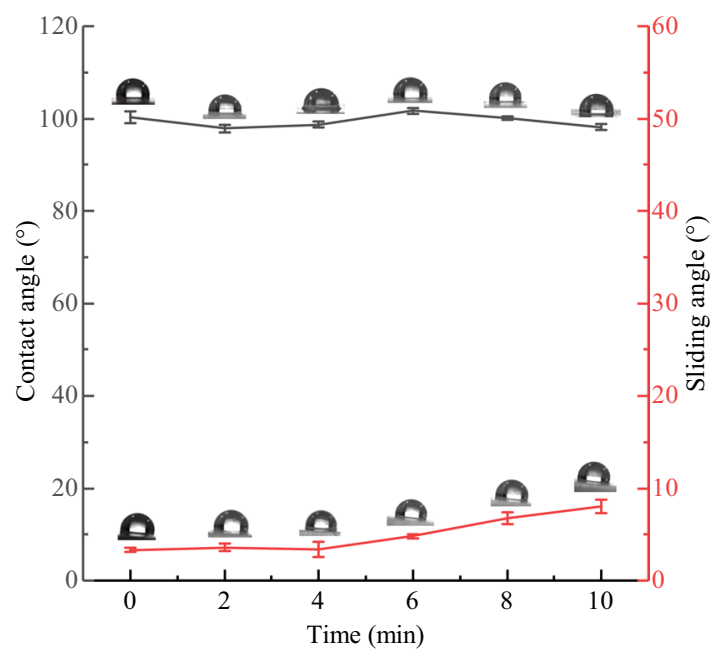

Fig. 4 a Oil loss of samples at different spin rates. b CAs and SAs of SLIPS with different $\mathrm{pH}$ values. $\mathbf{c}$ CAs and SAs of SLIPS after immersion in $0.1 \mathrm{M} \mathrm{HCl}$ solution for different time. d CAs and SAs of SLIPS after immersion in $0.1 \mathrm{M} \mathrm{NaOH}$ solution for different time

corroded by acidic and alkaline solution. It is clear that the surface damage was more severe after soaking in $\mathrm{HCl}$ solution. Obviously, the SHS cannot effectively resist the corrosion of the substrate by acid or alkali solution. On the contrary, as shown in the Fig. 4, the CAs of the SLIPSs did not change much after soaking in the solution. With the increase of immersion time, the SAs of the SLIPSs increases gradually, but the overall SAs were less than $10^{\circ}$. It indicates that the oil film on the SLIPS had certain resistance to the corrosion of acid and alkali solution in a short time. In short, the SLIPS is protected by the oil film, so it has better chemical and mechanical stability than SHS.

\subsection{Anti-corrosion Ability}

To study the corrosion resistance of the SLIPS, electrochemical corrosion tests were carried out in $\mathrm{NaCl}$ solution and compared with SHS and Bare. The potential polarization curves were obtained by the assistance of a three-electrode electrochemical workstation, and the corrosion current (Icorr) density and corrosion potential (Ecorr) were obtained via the Tafel extrapolation method. The data obtained from the electrochemical corrosion test were selected to plot the curve, and the results were shown in Fig. 5. As shown in Table 2, the corrosion potential (Ecorr) and corrosion current (Icorr) density of the group were displayed. The Icorr and Ecorr of Bare, SHS and SLIPS were $7.871 \times 10^{-6} \mathrm{~A}$, $3.585 \times 10^{-6} \mathrm{~A}, 1.247 \times 10^{-6} \mathrm{~A}$ and $-0.752 \mathrm{~V},-0.736 \mathrm{~V}$, $-0.684 \mathrm{~V}$, respectively. Generally, samples with superior corrosion resistance have higher corrosion potential (Ecorr) and lower corrosion current (Icorr) [33]. Therefore, this data indicate that SHS is superior to Bare, while SLIPS is better than SHS in corrosion resistance. It should be noted that low current density comes from the oil film, which makes the 


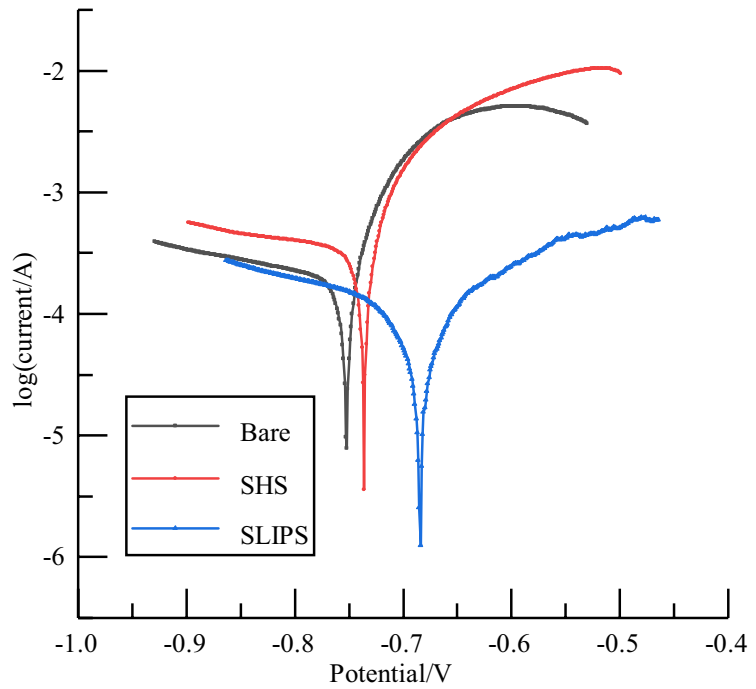

Fig. 5 Electrochemical corrosion tests of the Bare, SHS and SLIPS

Table 2 Corrosion characteristics of the samples in $3.5 \mathrm{wt} \% \mathrm{NaCl}$ solution

\begin{tabular}{ll}
\hline & $3.5 \mathrm{wt} \% \mathrm{NaCl}$ solution \\
\hline Samples & Ecorr (V) Icorr (A) \\
Bare & $-0.7527 .871 \times 10^{-6}$ \\
SHS & $-0.7363 .585 \times 10^{-6}$ \\
SLIPS & $-0.6841 .247 \times 10^{-6}$ \\
\hline
\end{tabular}

SLIPS have excellent corrosion resistance. The oil film insulates the contact between the substrate and the $\mathrm{NaCl}$ solution to protect the Al alloy. The result showed that lubricant has good physical barriers for protecting the Al alloy substrate from corrosion.

\subsection{Anti-fouling and Anti-icing}

In our daily life, the surface with antifouling ability has a huge impact, and it can improve the application range of materials [34]. Here, to compare the anti-fouling capability of the samples, they were immersed in comment different liquids (including coffee, tea, milk and cola) for $10 \mathrm{~s}$ then removed. It is noteworthy that all the liquid used for the test is thoroughly stirred to uniform. The test results are shown in Figs. 6a-d, after soaking in coffee, some liquid remains on the Bare, and the SHS can still be kept clean due to the water repellency (Fig. S6). Unexpectedly, the SLIPS can also keep clean after soaking in coffee (Fig. 6). The same effect was found in tea, milk and cola tests, the SHS and the SLIPS remained as clean as at the beginning while the surface of Bare had some residues (Figs. 6b-d). Remarkably, because of the existence of oil film, the SLIPS can repel most liquids with high surface tension, and the liquid can slide readily. In short, the SLIPS has slippery and hydrophobic properties, and liquid is not easy to remain on its surface, so it reveals fine anti-fouling performance. And the SLIPS is superior to SHS in the above-mentioned performances, thus, SLIPS may be used in anti-pollution in various industries in the future, such as construction, shipbuilding, automobile manufacturing, and so on.

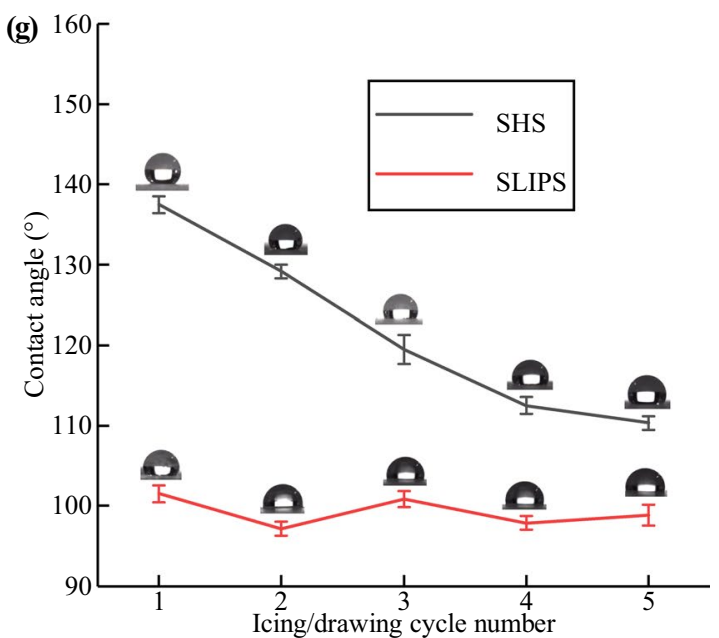

surface of the samples (Bare, SHS and SLIPS) after a long period of freezing. e CAs of SHS and SLIPS after freezing and drawing with different cycles in the anti-icing test
Fig. 6 A Series of anti-fouling tests testified the self-cleaning ability of the SLIPS (left) and SLIPS (right) immersed in a coffee, $\mathbf{b}$ tea, c milk and d cola. e The adhesion of different samples (Bare, SHS and SLIPS) to the ice surface when water freezes. $\mathbf{f}$ Changes in the 
Moreover, ice accretion has a serious impact on the usage life of equipment and the application of materials, resulting in huge disaster and economic losses [35]. Therefore, improving the ice resistance of materials is also a hot topic for researchers. Herein, the anti-icing performances of Bare, SHS, and SLIPS were tested. Under the condition of $-10^{\circ} \mathrm{C}, 15 \mathrm{~mL}$ water was injected into the culture dish, and then put the treated sample surface in contact with the water surface until the water freezes. The adhesion effect between the sample and the ice surface was compared. As shown in the Fig. 6e, Bare and HSH were firmly adhered to the ice surface, while SLIPS was easier to remove from the ice surface than them. The reason for this phenomenon may be that under low temperature conditions, the moisture adhering to the solid surface instantly freezes and sticks to the ice surface. However, SLIPS insulates the contact between water and the substrate due to the existence of the oil film, and the water molecules on the oil film are not easy to form ice nuclei and the ice crystals grow slowly, making it difficult to adhere to the ice surface $[36,37]$. The CAs during freezing and drawing cycles were measured and reflected in Fig. $6 \mathrm{~g}$. In the icing/drawing test, due to the destruction of SHS and the lubricant oil loss of SLIPS, they all lost their low sliding angle. The CA of SHS drops to about $110^{\circ}$ after five cycles for icing/stretching, while SLIPS can maintain a relatively stable CA due to the protection of the oil film. Furthermore, the petri dish was left in the refrigerator overnight, after a long time of freezing, there was almost no change on the slips, but slight damage on Bare and SHS (Fig. 6f). Thence, there is no doubt that SLIPS shows better anti-icing than Bare and SHS.

\section{Conclusion}

In summary, the multi-functional SLIPS have been constructed on Al alloy by a simple hydrothermal method, stearic acid modification and dimethyl silicone oil lubrication, successively. The experimental results showed that the three main factors of SLIPS were surface roughness, low surface energy and infused lubricant oil. The SLIPS based on $\mathrm{Al}$ alloy not only revealed significant hydrophobicity, but also has low slip angle, on which water droplets can slide at $5^{\circ}$. In addition, the stability of the SLIPS was also attractive. In the physical and chemical properties experiments, $43.06 \%$ of the lubricating oil can still be stored on the SLIPS after high shearing force, and it can maintain a low sliding angle after strong acid/alkali immersion.

Furthermore, the excellent anti-corrosion, anti-fouling and anti-icing performance of SLIPS are of great help to prolonged the service life of $\mathrm{Al}$ alloys and expand their practical application range. Moreover, SLIPS has broad application prospects in the fields of automobile, shipbuilding, architecture and so on. It is hoped that this paper can provide some reference for expand the practical application range of SLIPS.

Supplementary Information The online version contains supplementary material available at https://doi.org/10.1007/s42235-021-00124-6.

Acknowledgements This work was financially supported by the National Natural Science Foundation of China (no. 51735013 and 51905520) and the Pre-Research Program in National 14th Five-Year Plan (grant number 61409230614).

\section{Declarations}

Conflict of interests The authors declare that they have no known competing financial interests or personal relationships that could have appeared to influence the work reported in this paper.

Open Access This article is licensed under a Creative Commons Attribution 4.0 International License, which permits use, sharing, adaptation, distribution and reproduction in any medium or format, as long as you give appropriate credit to the original author(s) and the source, provide a link to the Creative Commons licence, and indicate if changes were made. The images or other third party material in this article are included in the article's Creative Commons licence, unless indicated otherwise in a credit line to the material. If material is not included in the article's Creative Commons licence and your intended use is not permitted by statutory regulation or exceeds the permitted use, you will need to obtain permission directly from the copyright holder. To view a copy of this licence, visit http://creativecommons.org/licenses/by/4.0/.

\section{References}

1. Zuo, Z. P., Liao, R. J., Guo, C., Yuan, Y., Zhao, X. T., Zhuang, A. Y., \& Zhang, Y. Y. (2015). Fabrication and anti-icing property of coral-like superhydrophobic aluminum surface. Applied Surface Science, 331, 132-139.

2. Zheng, S. L., Li, C., Fu, Q. T., Li, M., Hu, W., Wang, Q., Du, M. P., Liu, X. C., \& Chen, Z. (2015). Fabrication of self-cleaning superhydrophobic surface on aluminum alloys with excellent corrosion resistance. Surface and Coatings Technology, 276, $341-348$

3. Teisala, H., Tuominen, M., \& Kuusipalo, J. (2014). Superhydrophobic coatings on cellulose-based materials: Fabrication, properties, and applications. Advanced Materials Interfaces, 1, 1300026.

4. Ovari, F., Tomcsanyi, L., \& Turmezey, T. (1988). Electrochemical study of the pitting corrosion of aluminum and its alloys. 1 . Determination of critical pitting and protection potentials. Electrochimica Acta, 33, 323-326.

5. Wang, F. Y., \& Guo, Z. G. (2018). Insitu growth of durable superhydrophobic $\mathrm{Mg}-\mathrm{Al}$ layered double hydroxides nanoplatelets on aluminum alloys for corrosion resistance. Journal of Alloys and Compounds, 767, 382-391.

6. He, Z. H., Zeng, Y. W., Zhou, M. M., Min, Y. L., Shen, X. X., \& Xu, Q. J. (2021). Superhydrophobic films with enhanced corrosion resistance and self-cleaning performance on an $\mathrm{Al}$ alloy. Langmuir, 37, 524-541.

7. Li, X. W., Zhang, L., Shi, T., Zhang, C. W., \& Zhang, L. C. (2019). Facile preparation of superhydrophobic structures on Al alloys surfaces with superior corrosion resistance. Materials and Corrosion, 70, 558-565. 
8. Zhao, S. Y., Tie, L., Guo, Z. G., \& Li, J. (2020). Water deteriorates lubricating oils: Removal of water in lubricating oils using a robust superhydrophobic membrane. Nanoscale, 12, 11703-11710.

9. Zhang, X. M., Fu, F., Gao, X. M., \& Hou, X. F. (2019). Magnetically driven superhydrophobic polyurethane sponge for high efficiency oil/water mixtures separation. Journal of Bionic Engineering, 16, 38-46.

10. Liu, G. Y., Yuan, Y., Zhou, J., Jiang, Y. D., \& Wang, L. (2020). Anti-frosting/anti-icing property of nano- $\mathrm{ZnO}$ superhydrophobic surface on $\mathrm{Al}$ alloy prepared by radio frequency magnetron sputtering. Materials Research Express, 7, 026401.

11. Sarshar, M. A., Song, D., Swarctz, C., Lee, J., \& Choi, C. H. (2018). Anti-icing or deicing: Icephobicities of superhydrophobic surfaces with hierarchical structures. Langmuir, 34, 13821-13827.

12. Liu, W. L., Chen, H. F., Shen, Y. Z., \& Wu, Z. W. (2019). Facilely fabricating superhydrophobic resin-based coatings with lower water freezing temperature and ice adhesion for anti-icing application. Journal of Bionic Engineering, 16, 794-805.

13. Zhang, B. B., Zhu, Q. J., Li, Y. T., \& Hou, B. R. (2018). Facile fluorine-free one step fabrication of superhydrophobic aluminum surface towards self-cleaning and marine anticorrosion. Chemical Engineering Journal, 352, 625-633.

14. Yang, Z., Liu, X. P., \& Tian, Y. L. (2019). Hybrid laser ablation and chemical modification for fast fabrication of bio-inspired super-hydrophobic surface with excellent self-cleaning, stability and corrosion resistance. Journal of Bionic Engineering, 16, $13-26$.

15. Cheng, M. J., Song, M. M., Dong, H. Y., \& Shi, F. (2015). Surface adhesive forces: A metric describing the drag-reducing effects of superhydrophobic coatings. Small (Weinheim an der Bergstrasse, Germany), 11, 1665-1671.

16. Nguyen, T. P. N., Brunet, P., Coffinier, Y., \& Boukherroub, R. (2010). Quantitative testing of robustness on superomniphobic surfaces by drop impact. Langmuir, 26, 18369-18373.

17. Chen, X. S., Wen, G., \& Guo, Z. G. (2020). What are the design principles, from the choice of lubricants and structures to the preparation method, for a stable slippery lubricant-infused porous surface? Materials Horizons, 7, 1697-1726.

18. Bohn, H. F., \& Federle, W. (2004). Insect aquaplaning: Nepenthes pitcher plants capture prey with the peristome, a fully wettable water-lubricated anisotropic surface. Proceedings of the National Academy of Sciences of the United States of America, 101, 14138-14143.

19. Wong, T. S., Kang, S. H., Tang, S. K. Y., Smythe, E. J., Hatton, B. D., Grinthal, A., \& Aizenberg, J. (2011). Bioinspired selfrepairing slippery surfaces with pressure-stable omniphobicity. Nature, 477, 443-447.

20. Xiang, T. F., Zhang, M., Sadig, H. R., Li, Z. C., Zhang, M. X., Dong, C. D., Yang, L., Chan, W. M., \& Li, C. (2018). Slippery liquid-infused porous surface for corrosion protection with selfhealing property. Chemical Engineering Journal, 345, 147-155.

21. Shi, Z. Q., Xiao, Y. M., Qiu, R., Niu, S. Y., \& Wang, P. (2017). A facile and mild route for fabricating slippery liquid-infused porous surface (SLIPS) on CuZn with corrosion resistance and self-healing properties. Surface and Coatings Technology, 330, 102-112.

22. Sun, Y. H., \& Guo, Z. G. (2020). Novel and cutting-edge applications for a solvent-responsive superoleophobic-superhydrophilic surface: Water-infused omniphobic surface and separating organic liquid mixtures. Chemical Engineering Journal, 381, 122629.

23. Feng, R., Xu, C., Song, F., Wang, F., Wang, X. L., \& Wang, Y. Z. (2020). A bioinspired slippery surface with stable lubricant impregnation for efficient water harvesting. ACS Applied Materials and Interfaces, 12, 12373-12381.

24. Li, H., Wei, H. Y., Zou, X. Y., Wang, C. Y., Gao, Q., Li, Q., Liu, Q. Z., \& Zhang, J. F. (2020). Copper-based nanoribbons fabricated on a copper substrate by a liquid-solid reaction and their corrosion performance. Materials Chemistry and Physics, 246, 122839.

25. Cao, Y. H., Zheng, D. J., Li, X. L., Lin, J. Y., Wang, C., Dong, S. G., \& Lin, C. J. (2018). Enhanced corrosion resistance of superhydrophobic layered double hydroxide films with long-term stability on Al substrate. ACS Applied Materials and Interfaces, 10, 15150-15162.

26. Lei, J., \& Guo, Z. G. (2020). A fog-collecting surface mimicking the Namib beetle: Its water collection efficiency and influencing factors. Nanoscale, 12, 6921-6936.

27. Basu, M., Sinha, A. K., Pradhan, M., Sarkar, S., Negishi, Y., \& $\mathrm{Pal}, \mathrm{T}$. (2011). Fabrication and functionalization of $\mathrm{CuO}$ for tuning superhydrophobic thin film and cotton wool. The Journal of Physical Chemistry C, 115, 20953-20963.

28. Wang, Y., Zhang, D., \& Lu, Z. (2015). Hydrophobic Mg-Al layered double hydroxide film on aluminum: Fabrication and microbiologically influenced corrosion resistance properties. Colloids and Surfaces A: Physicochemical and Engineering Aspects, 474, $44-51$.

29. To, T. X. H., Truc, T. A., Duong, N. T., Vu, P. G., \& Hoang, T. (2012). Preparation and characterization of nanocontainers of corrosion inhibitor based on layered double hydroxides. Applied Clay Science, 67-68, 18-25.

30. Zhu, J. Y., Wan, H. R., \& Hu, X. F. (2016). A rapid one-step process for the construction of corrosion-resistant bionic superhydrophobic surfaces. Progress in Organic Coatings, 100, 56-62.

31. Golovin, K., Boban, M., Mabry, J. M., \& Tuteja, A. (2017). Designing self-healing superhydrophobic surfaces with exceptional mechanical durability. ACS Applied Materials and Interfaces, 9, 11212-11223.

32. Gao, X. Y., \& Guo, Z. G. (2018). Mechanical stability, corrosion resistance of superhydrophobic steel and repairable durability of its slippery surface. Journal of Colloid and Interface Science, 512, 239-248.

33. Xu, W. J., Song, J. L., Sun, J., Lu, Y., \& Yu, Z. Y. (2011). Rapid fabrication of large-area, corrosion-resistant superhydrophobic $\mathrm{Mg}$ alloy surfaces. ACS Applied Materials and Interfaces, 3, 4404-4414.

34. Kong, X. W., Zhang, J. Y., Xuan, Q., Lu, J. J., \& Feng, J. (2018). Superhydrophobic coating for antifouling of Chinese paintings. Langmuir, 34, 8294-8301.

35. Dou, R. M., Chen, J., Zhang, Y., Wang, X. P., Cui, D. P., Song, Y. L., Jiang, L., \& Wang, J. J. (2014). Anti-icing coating with an aqueous lubricating layer. ACS Applied Materials and Interfaces, 6, 6998-7003.

36. Gou, X. L., \& Guo, Z. G. (2020). Facile fabrication of slippery lubricant-infused $\mathrm{CuO}$-coated surfaces with different morphologies for efficient water collection and excellent slippery stability. Langmuir, 36, 8983-8992.

37. Fan, H. F., \& Guo, Z. G. (2020). Robust multi-functional slippery surface with hollow $\mathrm{ZnO}$ nanotube structures. New Journal of Chemistry, 44, 15483-15491.

Publisher's Note Springer Nature remains neutral with regard to jurisdictional claims in published maps and institutional affiliations. 